\title{
Conference Summary: The Demise of Spherical and Stationary Winds
}

\author{
Immo Appenzeller ${ }^{1,2}$ \\ 1 Landessternwarte, Königstuhl, D-69117 Heidelberg, Germany \\ 2 Max-Planck-Institut für Astronomie, Königstuhl, D 69117 Heidelberg, Germany
}

\begin{abstract}
The observational evidence and the theoretical work presented at this colloquium demonstrate that significant non-spherical effects and time variations are very common phenomena in the winds from hot stars. Hence, the assumptions of spherical symmetry and stationarity, while highly successful for the theory of stellar structure, appear to be inadequate for describing and understanding the winds of hot stars. This conclusion has significant consequences for the appearance and evolution of hot stars.
\end{abstract}

\section{Introduction}

In view of the huge distances of our research objects stellar astrophysics has been a surprisingly successful branch of science. Today the interiors of the Sun (and of main-sequence stars about anywhere in the Universe) are in many aspects better understood than the deep interior of our Earth, and many details of stellar atmospheres can be modeled more reliably than the behavior of the atmosphere in which we live.

Much of this success has been due to the fact that the basic physics of stars is relatively simple. In most cases their matter can be described by the equation of state of an ideal gas. Stellar structure can be approximated very well assuming spherical symmetry. And although all stars evolve with time, the evolutionary time scale is so much longer than the dynamic time scale that static or stationary models provide excellent approximations.

Since about 70 years it has been known that luminous hot stars tend to lose mass. Later it became clear that late-type stars like our Sun also have stellar winds and that stellar mass loss is a rather common phenomenon occurring in all regions of the HR diagram. In view of the simplicity and the success of the spherical and static stellar structure models it was natural to start modeling stellar winds with spherical and stationary configurations too. Although direct observations of Planetary Nebulae, Nova and Supernova remnants, wind-blown IS bubbles, and X-ray observations of the solar corona showed conspicuous deviations from spherical symmetry, the notion of spherically symmetric and stationary winds survived, mainly due to our lack of knowledge on the real wind geometries.

At this conference much additional evidence for non-spherical and nonstationary winds was reported. Moreover, we learned that major deviations 
from spherical symmetry and stationarity follow naturally from realistic wind theories. Hence, IAU Colloquium 169 may one day be remembered as the meeting where it was realized that the assumptions of spherical symmetry and stationarity (while very successful for stellar interiors) is fundamentally inadequate for stellar winds. In the following I shall try to summarize the results which were presented at this Colloquium and which lead us to this conclusion.

\section{Probing the Wind Geometry}

\subsection{Observational Progress}

The occurrence of stellar winds was initially inferred from spectroscopic data (Beals, 1930). Spectroscopy also provided the first hints for deviations from spherically symmetric flows, and this technique continues to be one of the main information sources on the wind geometries. Perhaps the most impressive data sets shown at this meeting were the extensive spectroscopic time series of $\mathrm{O}$ stars, BA supergiants and Be stars obtained with IUE and with dedicated ground-based instruments, such as HEROS, and described elsewhere in this volume by A.W. Fullerton, Andreas Kaufer, Geraldine Peters, Stanislav Štefl, and Thomas Rivinius. All these observations resulted in convincing evidence for rotational modulations of the wind spectra, proving conclusively that the observed winds are not spherically symmetric and, in fact, not even azimuthally symmetric. Various plausible models for wind geometries have been derived and presented at this meeting. However, the discussion also showed that, while spectroscopic time series can prove the presence of asymmetries conclusively, the derivation of the exact wind structure from spectroscopic data is complex and much more difficult.

A more direct method to prove the presence of deviations from spherical symmetry is polarimetry. This technique has been used to investigate flattened objects and circumstellar disks since more than three decades (cf. e.g. Appenzeller 1965, Appenzeller and Hiltner 1967). But major progress in this field was achieved only during the past decade with the development of improved instrumentation, better models and the introduction of spectropolarimetry. This progress was demonstrated impressively at this meeting by the reports and posters presented by Karin Bjorkman, Mário Magalhães, Cláudia Vilega Rodrigues, and R.V. Yudin. But, as analyzed beautifully in John Brown's talk, inferring the geometric details from polarimetry and spectropolarimetry alone requires caution, and a successful fit to a simple model does not guarantee a correct derivation for the underlying geometry. Therefore, polarimetric observations cannot fully replace direct high angular resolution imaging.

As expected some of the key results in the field of high-resolution direct imaging of winds presented at this meeting came from the HST (cf. the corresponding contributions by Antonella Nota, Regina Schulte-Ladbeck, and 
others to this volume). There were also interesting new radio data (S. White) and - more surprisingly - FIR data from ISO (N. R. Trams et al.). That ISO (with its angular resolution strongly limited by the diffraction disk of its, expressed in wavelength, rather small aperture) should allow us to image the wind zones of stars appears unexpected on first glance. On the other hand, ISO records radiation from the cold matter. Hence ISO allows us to see the cool and dusty outermost regions of wind flows, which are sufficiently extended to be resolved with infrared satellites.

Among the most exciting results presented at this meeting were data obtained from optical interferometric imaging (F. Vakili and others). The data produced so far show clearly the wavelength dependence of the shape, orientation and size of the wind flows on a sub-arcsec scale. The next step obviously must be to obtain a more complete coverage of the $u-v$ plane and to reconstruct real images of the observed stellar winds. While this will require much additional development work, it was very encouraging to see real progress in this field. As a precaution it must be noted however, that for direct and interferometric imaging the length scales which can actually be resolved will always depend on the objects distance. In this respect indirect methods like polarimetry and spectroscopy have the advantage of working equally well for any distance. Hence all these techniques will remain important for future work.

\subsection{A Choice of Theories}

Non-spherical mass loss was inferred first for Be stars. Very soon a relation with the rotational velocity was discovered and rotation became the prime suspect for causing non-spherical winds (Sletteback 1949). That other physical mechanisms may (also) be involved became clear when strongly nonsymmetric winds were found in the (slowly rotating) B[e] supergiants (Zickgraf et al. 1985). Initially it appeared difficult to reconcile this result with the established theories of (radiation driven) winds of hot stars. Today there is no longer any doubt that through various mechanisms even a small amount of rotation can cause large asymmetries in radiation driven winds. (For details see the contributions by Jon Bjorkman, Joachim Puls, Henny Lamers and others to this volume). However, the lively discussion at this colloquium could not clarify whether rotationally induced compression of the gas in the equatorial plane or bi-stable winds (or a combination) are the main source of the observed asymmetries. The theory of wind compressed disks appears better developed at present, but seem to have problems reproducing the required wind parameters. No such problems seem to exist in the case of rotationally induced bi-stability, but this could simply be due to a lower stage of development of this theory. Finally, one has to keep in mind that both concepts are approximations. And magnetic fields (invoked by various authors) may complicate things further. 
Fortunately, the different theories predict significantly different wind geometries. Rotationally induced wind compression will result in geometrically thinner disks than bi-stable winds or magnetically supported disks. Hence future interferometric images of the wind geometry may well help us to identify the dominant mechanism unambiguously.

\section{Variable Winds}

Variability of luminous hot stars and of their winds has been observed since several decades. (Note that for the hot star where mass loss has been inferred first, P Cyg, variability had been discovered already in 1600, making P Cyg one of the first two stars - apart from Novae and $\mathrm{SNe}$ - for which variations were established). But only during the past ten years it became clear that wind variations is an omnipresent feature of these stars.

The variations may be minor (as in $\theta^{1}$ Ori $\mathrm{C}$ ) or dramatic (as in the LBVs). The small amplitude variations seem to be reasonably well understood as effects of rotation (as already discussed in Section 2.1), pulsations (see Section 4), or wind instabilities (see the contributions of A. Feldmeier and Stan Owocki to this volume). As pointed out by W.-R. Hamann and by Gloria Koenigsberger the formation of clumps seems to be a major cause of the variations in the dense winds of the Wolf-Rayet stars. On the other hand, the true physical origin of the dramatic large amplitude variations of the LBVs remains unclear. Although our empirical knowledge on the properties is fairly extensive by now (cf. Otmar Stahl's contribution) little progress has been reported concerning the theory of these large amplitude LBV variations. There exist since many years various suggestions for mechanisms (discussed already in the proceedings of IAU meetings Symposium 116 and IAU Colloquium 113). But these ideas have not been followed up by detailed model computations. Moreover, as pointed out by Roberta Humphreys at this meeting, the LBVs may well consist of at least two distinct classes with different physical variability mechanisms.

\section{Pulsations}

For many decades investigations of hot pulsating stars were restricted essentially to the $\beta \mathrm{Cep}$ (or $\beta \mathrm{CMa}$ ) stars, although the theorists had predicted since a long time that pulsational instability may be present in a much larger fraction of the blue part of the HR-diagram (see e.g. Osaki 1987). At this meeting pulsational instability of various modes (radial, non-radial, strange mode) was reported for about any effective temperature and luminosity where hot stars can be found (for details see the contributions to this volume by Henrichs, Baade, Osaki, Glatzel, Guzik, and Ødegaard). Also new at this meeting was the finding that beating effects between the many different nonradial modes could have important astrophysical consequences and perhaps 
play a major role for the mass loss from Be stars (as discussed in a contribution by Thomas Rivinius).

\section{Consequences of Non-radial and Non-stationary Winds}

\subsection{Evolutionary Effects}

An obvious consequence of winds from hot stars is the formation of wind blown bubbles, circumstellar nebulae, and sometimes cool and dusty disks. Progress in our understanding of these phenomena has been reported at this meeting (and is described in this volume) in the contributions of Mordecai Mac Low, Linda Smith et al. and R. Waters.

It is well known that stellar winds have a profound influence on the evolutionary tracks of massive stars in the HR diagram (cf. A. Maeder, G. Meynet, and N. Langer in this volume). Therefore, in all modern evolutionary calculations for massive stars mass loss has been taken into account in some semi-empirical way (see e.g. Meynet et al. 1994). However, all these calculations assumed for simplicity spherical and stationary mass loss. As pointed out at this meeting, more realistic assumptions may affect the evolutionary time scales and the computed luminosities significantly. New tracks will have to be calculated before a quantitative assessment of the expected differences can be carried out.

\subsection{Luminous Hot Stars as Distance Indicators}

With luminosities often surpassing those of globular clusters and of whole small galaxies LBVs can be observed and identified easily in many resolved extragalactic systems. Hence they are potentially very important extragalactic distance indicators, provided their brightness can be calibrated with sufficient accuracy.

Various methods to obtain such calibrations have been suggested in the literature. At this meeting two such methods have been discussed in some detail. R.-P. Kudritzki reported new results on the Wind Momentum Luminosity Relation (WMLR) method (Kudritzki et al. 1995), and Berhard Wolf and others discussed the use of the Amplitude-Luminosity Relation (ALR) of the LBVs (Wolf (1989)).

On first glance one might suspect that time variations and deviations spherical symmetry could compromise the accuracy of the WMLR method. However, as demonstrated in R.-P. Kudritzki's contribution, fortunately these effects influence the WMLR only to a minor extent. Hence the WMLR should remain one of the potentially most accurate and powerful procedure to determine the distance of resolved galaxies.

Wolf's ALR method, of course, is based on time variations, and in view of the statistical calibration should not be influenced much by non-spherical 
winds. Hence its potential should not be affected by the results discussed at this meeting. Comparing the WMLR and the ALR, the WMLR has the obvious advantage of giving accurate results from a single epoch observation, while the ALR requires observations at several observing epochs at uncomfortably large time intervals. On the other hand, being a purely photometric method the ALR in principle can reach much larger distances. It is somewhat surprising that not more use is being made of this method.

\section{Outlook}

Because of their short life expectancy luminous hot stars are relatively rare objects. Moreover, most galactic hot luminous stars are hidden from our view as they are strongly concentrated to the galactic plane and are obscured by the galactic dust clouds. Therefore, much of our knowledge about these objects is based on observations in other nearby galaxies, notably the Magellanic Clouds. On the other hand large telescopes and efficient instruments are needed to study extragalactic stars. Just at the time of the final preparations of IAU Colloquium 169 the ESO-VLT UT1, the first very large telescope in the southern hemisphere started its operations. Together with the already routinely operating two Keck telescopes and several comparable instruments close to completion or under construction these new facilities will make observations of extragalactic stars much easier and, with powerful new focal plane instruments such as FORS, much more economic. There is no doubt that these new generation of telescopes will greatly stimulate research in the area of hot luminous stars with all the techniques discussed at this colloquium.

One of unique features of the ESO VLT will be the possibility to combine the four unit telescopes and several smaller auxiliary instruments to a powerful optical interferometer, which promises major progress in the interferometric imaging of stellar envelopes.

I am somewhat sorry to note that in view of these new technical opportunities which will become available during the next months and years, it seems safe to predict that much of the observational data reported at IAU Colloquium 169 and much of what has been written down in this volume will soon become completely obsolete and replaced by much superior results. On the other hand, I am also convinced that the high scientific standard of this colloquium and the very lively discussions of this meeting will certainly have a significant impact on the research in our field during next years and that in this way our meeting may have contributed much to a productive and efficient use of the new large telescopes for stellar astrophysics.

\section{References}

Appenzeller, I. (1965): ApJ 141, 1390 
Appenzeller, I. and Hiltner, W.A. (1967): ApJ 149, 353

Beals, C.S. (1930): Pub. Dom. Ap. Obs. Victoria 4, 288

Kudritzki, R.-P., Lennon, Puls, J., (1995), in "Science with the VLT", J.R. Walsh and I.J. Danziger eds., Springer, Heidelberg 1994, p. 246

Meynet, G., Maeder, A., Schaller, G., Schaerer, D., Charbonnel, C. (1994): Astron. Astrophys. Suppl.103, 97

Osaki, Y. (1987), in "Instabilities in Luminous Early Type Stars", H.J.G.L.M. Lamers and C.W.H. de Loore eds., D. Reidel Publ. Co. , Dordrecht 1987, p. 39

Slettebak, A. (1949): ApJ 110, 498

Wolf, B., (1989), in Proc. IAU Coll. 113 on Physics of Luminous Blue Variables, K. Davidson, A.F.J. Moffat, H.J.G.L.M. Lamers eds., Kluwer, Dordrecht, p. 91. Zickgraf F.-J., Wolf, B., Stahl, O., Leitherer, C., Klare, G. (1985): Astron. Astrophys. 143, 421

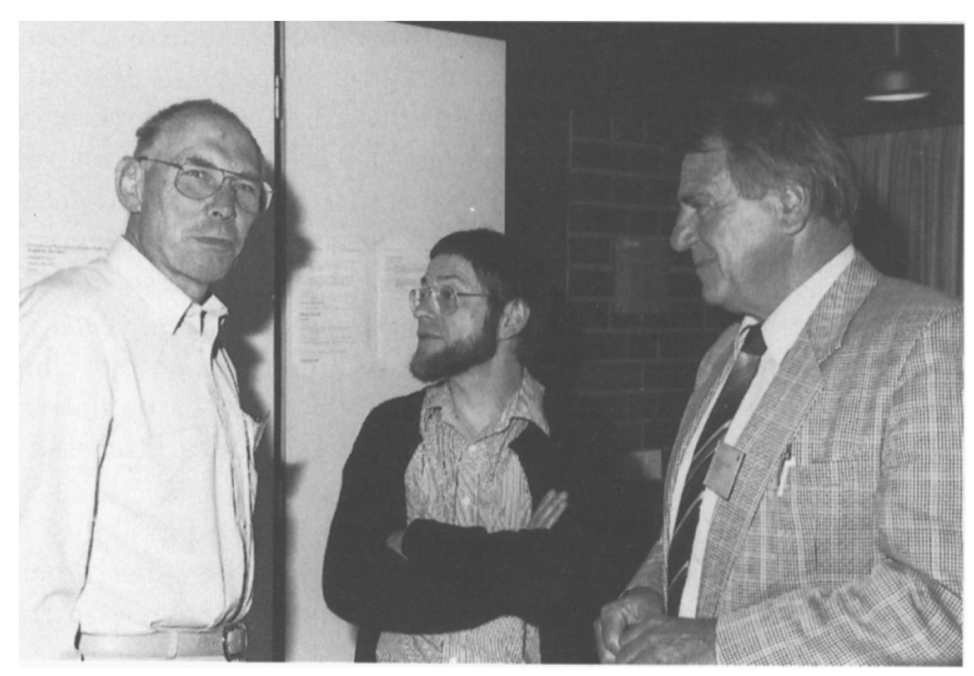

Immo Appenzeller, Ulrich Bastian and Bernhard Wolf 